\title{
More than meets the eye: age differences in the capture and suppression of oculomotor action
}

\author{
K. Richard Ridderinkhof ${ }^{1,2 *}$ and Jasper G. Wijnen ${ }^{1}$ \\ ${ }^{1}$ Amsterdam Center for the Study of Adaptive Control in Brain and Behavior, Department of Psychology, University of Amsterdam, Amsterdam, Netherlands \\ ${ }^{2}$ Cognitive Science Center Amsterdam, University of Amsterdam, Amsterdam, Netherlands
}

Edited by:

Jutta Kray, Saarland University,

Germany

Reviewed by:

Frini Karayanidis, University of

Newcastle, Australia

Guido P. H. Band, Leiden University,

Netherlands

${ }^{*}$ Correspondence:

K. Richard Ridderinkhof, Amsterdam

center for the study of adaptive control in brain and behavior.

Department of Psychology, University of Amsterdam, Roetersstraat 15, 1018 WB Amsterdam, Netherlands. e-mail: k.r.ridderinkhof@uva.nl
Salient visual stimuli capture attention and trigger an eye-movement toward its location reflexively, regardless of an observer's intentions. Here we aim to investigate the effect of aging (1) on the extent to which salient yet task-irrelevant stimuli capture saccades, and (2) on the ability to selectively suppress such oculomotor responses. Young and older adults were asked to direct their eyes to a target appearing in a stimulus array. Analysis of overall performance shows that saccades to the target object were disrupted by the appearance of a task-irrelevant abrupt-onset distractor when the location of this distractor did not coincide with that of the target object. Conditional capture function analyses revealed that, compared to young adults, older adults were more susceptible to oculomotor capture, and exhibited deficient selective suppression of the responses captured by task-irrelevant distractors. These effects were uncorrelated, suggesting two independent sources off age-related decline. Thus, with advancing age, salient visual distractors become more distracting; in part because they trigger reflexive eye-movements more potently; in part because of failing top-down control over such reflexes. The fact that these process-specific age effects remained concealed in overall oculomotor performance analyses emphasizes the utility of looking beyond the surface; indeed, there may be more than meets the eye.

Keywords: oculomotor capture, inhibitory control, saccades, aging, distributional analysis

\section{INTRODUCTION}

When our actions are determined by the environment rather than by a conscious plan to act, such actions are often called stimulusdriven or exogenous. In the attentional literature the additional term bottom-up is often used to describe the deployment of attention when governed by outside stimuli only. The term bottom-up refers to the fact that sensory information enters the brain via what are considered to be lower areas in a hierarchically organized brain. Stimulus-driven actions are guided directly by information sent up from perceptual areas toward areas involved in action selection and execution; action control areas are involved when such externally triggered actions are to be overruled by those that emanate from internal decisions (for a review see Ridderinkhof et al., 2011). Accordingly, the influence that comes from these control areas is often called top-down.

In this study we will investigate the relative strength of bottomup versus top-down processes in elderly compared to normal subjects. Specifically we will examine these processes with respect to oculomotor behavior. Distributional analysis will be used to assess the build-up of top-down control of eye-movements in an oculomotor capture task. This task, originally devised by Theeuwes et al. (1998), involves searching for a color singleton in a stimulus array that has six circles positioned on an imaginary circle around a central fixation point. Initially the array contains six identical circles until at a certain point in time five of the circles change color. The circle not changing color forms the target. Simultaneously with the color change an abrupt-onset distractor in the form of an extra circle is presented. Subjects are to react manually to the orientation of a line segment inside the target circle that is only recognizable when the eyes fixate on the correct circle directly. Theeuwes et al. (1998) found that even though in this task the abrupt-onset is irrelevant to the task, in many trials the eyes moved to it nonetheless. The automatic movement of the eyes toward an irrelevant onset distractor was termed oculomotor capture.

In a follow-up study these authors used the paradigm to investigate potential differences in the efficiency of attentional control between elderly subjects and young controls. Kramer et al. (1999) sought to answer whether age adversely affects our ability to negate bottom-up influences in attentional and oculomotor behavior. An increased level of distractibility has often been suggested to underlie performance deterioration with aging on a number of tasks (e.g., Rabbitt, 1965). Kramer et al. (1999) observed that young and older adults made comparable amounts of saccades to abruptonset distractors, indicating that both groups had equal difficulty controlling their oculomotor behavior. This was deemed a surprising finding, as previous research had shown that older adults exhibit poorer inhibitory control than young adults on a variety of different tasks (Zacks and Hasher, 1997), as well as a much higher percentage of misdirected saccades than young adults on the antisaccade task (for a critical review see, Nieuwenhuis et al., 2000). In a later study, using perceptually more salient (superluminant) abrupt-onset distractors, Kramer et al. (2000) did observe 
the expected age-related increase in saccade errors, giving rise to the hypothesis that as we age, we are increasingly susceptible to the disrupting effects of distractors, but only if we are sufficiently aware of those distractors.

We note that this analysis depends on the assumption that the percentage of misdirected saccades reflects inhibitory efficiency, and hence that this measure of saccade errors should reveal age differences in inhibitory control. However, such an analysis fails to take into account an interesting and relevant theoretical distinction between distinct neurocognitive processes. The susceptibility to oculomotor capture and the proficiency of inhibitory control over such capture, while both contributing to the percentage of misdirected saccades, can be argued to represent distinguishable processes, supported by different neural systems (for review see Ridderinkhof et al., 2011), and in principle be differentially sensitive to individual differences such as those related to aging. Thus, any pattern of age-related differences in eye-movements being misdirected to task-irrelevant onsets may pertain to differences in oculomotor capture among young and older adults, or to differences in inhibition, or both. That is, age effects may relate to increased susceptibility to oculomotor capture, or increased failure to suppress such capture, or both. We argue that a closer look at the data might shed light on this important issue.

\section{THE PRESENT STUDY}

In the current study our main interest lies in age differences in oculomotor capture and the process of active and intentional selective inhibition of such reflexive oculomotor responses. Stürmer et al. (2002) argued that such selective suppression is triggered by a conflict between the responses activated by bottom-up and topdown processes. When such a conflict is detected the response that was directly captured is selectively suppressed until the conflict is resolved.

An important assumption in these suppression theories is that the selective suppression takes some time to evolve (for a review see van den Wildenberg et al., 2010). This characteristic enables us to examine the effects of this process in reaction time (RT) distributions since suppression would differentially affect faster as opposed to slower responses. Negating irrelevant influences in a task like the oculomotor capture task is easier in the slowest responses since the system can take full advantage of selective suppression (Wijnen and Ridderinkhof, 2007). Likewise, since inhibition has not yet set in during the fastest responses, oculomotor capture by the irrelevant distractor is expressed in its purest form in the fastest responses. The development of suppression can be tracked by dividing collected RT data in multiple speed bins (for each experimental condition). Mean RT and accuracy can be computed for each of these bins and plotted against each other, resulting in conditional accuracy functions (CAFs). In the oculomotor capture task a direct eye-movement to the target is considered an accurate reaction. However saccades not going to the target do not necessarily land on the distractor. A representative picture of the incidence of oculomotor capture as a function of reaction speed can be obtained by creating conditional capture functions (CCF) which plot the percentage of eye-movements to the distractor against saccade RT (SRT; Wijnen and Ridderinkhof, 2009).
In the current study we intend to apply CCF analysis techniques in an oculomotor capture task with a design similar to Kramer et al. (2000). The oculomotor capture task was slightly modified to allow us to chart congruence effects. An identical stimulus configuration with six equi-spaced circles arranged in an imaginary circle around a central fixation point was used. The onset appeared as a slightly larger and superluminant circle around one of the six circles rather than appearing in between two of the circles. On half of all trials the onset appeared around the circle that also figured as the target singleton creating a congruent situation. On the other trials the distractor appeared around one of the other circles forming an incongruent trial.

While we expect to replicate the global pattern of results found by Kramer et al. $(1999,2000)$, we expect additionally that if agerelated deficiencies result from increased oculomotor capture, then this will be manifested in an effect of age on the fastest quantiles of the CCF. The first quantile of the CCF consists of very fast saccades, executed at a moment where selective suppression of responses based on bottom-up activation has not yet had time to develop. Consequently any differences between the experimental groups found on this first quantile are likely a result of attentional and oculomotor capture processes rather than processes related to oculomotor inhibition.

If however the difficulties encountered by the older group are a function of less efficient selective suppression, then we expect differences between the older and younger groups to be found instead on the slopes of the CCFs. The development of selective suppression is expected to reduce the percentage of eye-movements to the distractor on incongruent trials with slower RTs, resulting in CCFs that reach an asymptote toward the slower tail of the RT distribution. Sharper reductions in the amount of captured saccades toward the distractor are anticipated when selective suppression is strong. Therefore if younger subjects are better able to selectively suppress saccades to the distractor, this will translate to CCFs reaching asymptote faster, and thus steeper slopes between CCF levels at fast and slower quantiles.

\section{MATERIALS AND METHODS PARTICIPANTS}

Twenty undergraduate students (13 female; mean age: 20.65 , SD: 1.90, min: 19, max: 25 ) and 20 seniors (17 female; mean age: 64.25 , SD: 3.63 , min: 56, max: 69) took part in the experiment. Students were recruited at the University of Amsterdam and obtained course credits for their participation. Seniors were recruited using advertisements in local house-to-house magazines and newspapers. This group was awarded money for their participation. Subjects were tested individually in a quiet and dimly lit university chamber. All participants had normal or corrected-to-normal vision.

\section{STIMULI AND APPARATUS}

Participants were seated in a comfortable chair at a distance of $55 \mathrm{~cm}$ in front of a computer screen on which stimuli where shown against a black background. A chinrest stabilized the subject's head during the experiment to minimize movement artifacts in the eye-tracker data. Two response button boxes were placed within arm-reach on both sides of the chin rest. Eye-movements were recorded using an infrared-based iView eye-tracker (SMI, Berlin, 
Germany) with $50-\mathrm{Hz}$ temporal resolution and $\mathrm{a}<0.1^{\circ}$ spatial resolution.

A small white cross (subtending a visual angle of $0.7 \times 0.7$ ) served as a fixation point during the experiment. Each trial started with the presentation of the fixation point only. After $300 \mathrm{~ms}$ six equi-spaced gray circles (diameter $3.2^{\circ}$ ), containing figure-eight shapes, were presented on an imaginary circle (diameter $9.6^{\circ}$ ), around the fixation point. After $2200 \mathrm{~ms}$ all but one of the circles changed to an orange color and all figure-eight pre-masks changed into letters. The letter in the circle that did not change color (target circle) changed into a $\mathrm{C}$ or a reversed $\mathrm{C}$. Simultaneously with the color-change an additional larger dashed circle contour appeared (diameter $3.9^{\circ}$ ) around one of the six circles as a distractor (see Figure 1). In addition the fixation cross darkened to match the luminance of all other objects on the screen $\left(18 \mathrm{~cd} / \mathrm{m}^{2}\right)$. On $50 \%$ of trials the distractor circle surrounded the target circle (congruent trial). On all other trials (incongruent) the distractor circle surrounded one of the circles that changed color (non-target circles). The distance between the distractor and target circles was maximal on $50 \%$ of incongruent trials (three circles). On other incongruent trials this distance was minimal (one circle clockwise or anticlockwise). This display remained on screen for $3000 \mathrm{~ms}$ or until one of the response buttons was pressed. A black empty screen was then shown for $300 \mathrm{~ms}$ after which the next trial started. Distractor circle luminance could be either equiluminant to the other stimuli (gray circles, color-changed orange circles, pre-masks, letters; $\left.18 \mathrm{~cd} / \mathrm{m}^{2}\right)$ or superluminant to these objects $\left(68 \mathrm{~cd} / \mathrm{m}^{2}\right)$.

\section{PROCEDURE AND DESIGN}

All procedures were approved by a local ethics committee and conducted in accordance with relevant laws and institutional guidelines. Written informed consent was obtained from each individual prior to participation. The experiment involved one session lasting approximately $2 \mathrm{~h}$. After informed consent was obtained subjects completed a health questionnaire. Subjects were then tested for

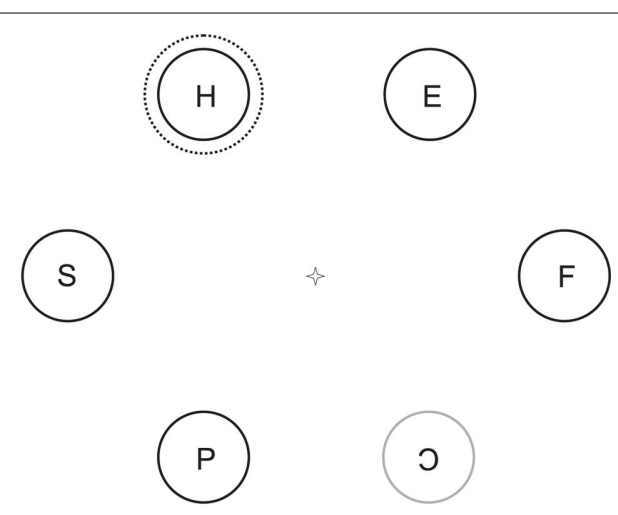

FIGURE 1 | A possible incongruent experimental trial after target and distractor presentation. The five black circles represent non-targets that have just changed color from gray to orange. The single gray circle did not change color and forms the target. The dashed circle appears simultaneously with the target and forms the distractor. The distractor can be either superluminant or equiluminant to all other stimuli. In this trial subjects should make a saccade to the bottom right circle and respond with the left button. reading acuity and color vision using Ishihara color plates (Ishihara, 1992). Subsequently subjects were asked to take place in front of the eye-tracker and stimulus presentation screen and calibration started. Calibration targets appeared at locations which would later be occupied by the experimental stimuli and the fixation cross. Subjects fixated four series of seven calibration targets. After the experimenter had verified that all instructions were well understood participants performed one practice block (without distractors) to familiarize them with the task and procedure. Subjects then proceeded with the experimental phase, which contained six identical blocks containing 144 trials each. Before the first block of the experimental phase started, subjects were warned about objects appearing that had not been mentioned in the instructions nor had been present in practice blocks. Distractors were equiluminant for half of the subjects in each age groups, and superluminant for the other half. Subjects were told that anything not covered by the instructions was irrelevant to the task and were instructed to try to ignore any "new" objects.

\section{EYE-TRACKER DATA HANDLING}

Saccade RTs were extracted offline from the eye-tracker data. SRTs were defined as the time at which the velocity of the eye-movement exceeded $100 \%$ s. For saccade accuracy purposes the visual field was divided into seven regions of interest (ROIs), one for the fixation point and one for each of the six stimulus locations. Each eyetracker data sample was categorized according these ROIs. When speed criteria were met the saccade goal was defined as the ROI of the first sample that was outside of the fixation ROI. If this ROI matched the target location the trial was treated as correct. In all other cases the trial was defined as an error, except when the eye did not leave fixation or velocity criteria where not met within $1000 \mathrm{~ms}$ after target presentation, in which case the trial was excluded from analysis as a miss $(0.76 \%)$. If more than six samples $(120 \mathrm{~ms})$ in a time window stretching from target appearance to manual response were lost then the trial was excluded due to movement artifact/blink $(0.70 \%)$. Trials were also excluded if the subject was not looking at the fixation cross at the time of target presentation (sample outside of fixation ROI; $4.73 \%$ ) or when the subject looked away from fixation in the first $100 \mathrm{~ms}$ after target appearance (fast guess; $1.44 \%$ ).

Drift was corrected by gathering positional data from all eyetracker samples recorded at the time of target presentation for each trial within a block (before fast guess removal but after artifact rejection). A moving average of \pm 15 trials was computed for each of the trials. This data was used to correct the raw positional eye-tracker data before assigning ROIs to data samples.

Outliers were removed from the data using a recursive procedure adopted from McCormick (1997; cf. Wijnen and Ridderinkhof, 2007), which involved temporarily removing the fastest and slowest RTs from each condition for each participant. After removal the mean and SD for the remaining data was calculated. If either of the two removed data-points fell outside an interval bounded by 4 SDs from the mean, that data-point was removed permanently. If the data-point fell within the interval it was returned to the data set. This procedure continued until no more data-points were removed permanently. By using this procedure $1.28 \%$ of the data was removed as outlier. 


\section{STATISTICAL ANALYSIS}

Trials classified as misses, eyes-not-on-fixation at the time of target presentation, movement artifact/blink, fast guess, or outlier, were dropped from all further analyzes. Oculomotor performance on the task was analyzed by calculating mean SRT and accuracy (percentage of eye-movements to the target) for each subject and for each condition. These values were entered as dependent variables in two separate GLM repeated-measures ANOVAs with congruence (within-subjects) and age and luminance (between-subjects) as independent variables. A similar set of ANOVAs was used to investigate manual performance (here accuracy consisted of the percentage of manual reactions in which the correct response button was pressed).

The degree of oculomotor capture was investigated by computing the percentage of eye-movements to the distractor. Since we expected a build-up of selective suppression with increased SRTs the percentage of saccades to the distractor was investigated as a function of reaction speed. To examine this, we applied a distribution analysis based on CCFs (see Wijnen and Ridderinkhof for detail). In short, SRTs for each subject were rank-ordered for each congruence condition. Both correct and incorrect trials were included. The SRTs were then divided into five equal-size speed bins, thus comprising vincentized CDFs (Vincent, 1912). For each of the bins the percentage of trials where the fist eye-movement went to the distractor was calculated. This percentage was plotted against mean SRT for each bin resulting in a CCF. The steepness of the slopes was quantified by subtracting the difference between two consecutive CCF data-points on the $y$-axis (percentage of capture) and dividing this value by the difference between two consecutive data-points on the $x$-axis (mean SRT). Slope steepness was subsequently entered in a repeated-measures ANOVA as a dependent variable with congruence and segment (within-subjects) and age and luminancy (between-subjects) as independent variables. The variable Segment refers to the quantiles from which the slope steepness was calculated, so that segment 1 was derived from the differences between the first and second quantile, segment 2 from the second and third, and so forth. To examine whether prior differences existed in oculomotor capture prior to the advent of selective oculomotor response suppression a second ANOVA was conducted with the percentage of saccades to the distractor in the first quantile as the dependent variable and the segment variable omitted.

\section{Conditional capture: correlations between bottom-up capture and top-down inhibition}

The extent to which oculomotor capture and oculomotor inhibition represent independent processes, a Pearson correlation analysis was conducted on the percentage of eye-movements to the incongruent distractor on the first quantiles of the CCFs with the slopes of the first segment of the CCFs. This analysis was conducted for each age group separately, to prevent age-related co-variance from artificially influencing the process correlations.

\section{RESULTS}

When necessary, $p$ values were corrected using the GreenhouseGeisser adjustment of degrees of freedom. The between-subjects factor luminance (equi- versus superluminant) did not interact with the effects of other factors, except in one higher-order interaction, and therefore is not mentioned except for that interaction.

\section{OVERALL OCULOMOTOR SPEED AND ACCURACY}

Figure 2 shows mean SRTs and overall accuracy for all groups as function of congruence. Compared to young participants, older adults were considerably slower $[F(1,36)=22.39, p<0.001]$ and less accurate $[F(1,36)=200.37, p<0.001]$ in fixating the target. All participants responded faster $[F(1,36)=102.15, p<0.001]$ and more accurate $[F(1,36)=114.60, p<0.001]$ on congruent compared to incongruent trials. The effect of congruence was more pronounced for older compared to young participants for SRT $[F(1,36)=14.12, p=0.001]$, but not for accuracy $[F(1,36)=2.70$, $p=0.109]$.

\section{OVERALL OCULOMOTOR CAPTURE}

On incongruent trials the percentage of initial eye-movements going to the distractor is a somewhat more accurate measure of oculomotor capture than accuracy (eye-movements to the target). As expected, trial congruence affected the percentage of eyemovements going to the distractor $[F(1,36)=357.82, p<0.001$; see Figure 3] as subjects were more likely to avoid the distractor location on incongruent trials. Older participants were less proficient in ignoring the distractor on incongruent trials, and benefited less when target and distractor coincided on congruent trials resulting in an interaction between congruence and age $[F(1,36)=15.95, p<0.001]$.

\section{DISTRIBUTIONAL ANALYSIS OF OCULOMOTOR PERFORMANCE}

Figure 4 shows the percentage of saccades to the distractor as a function of response speed. As can be seen from the graph fast reactions have a tendency to be captured by the distractor on both congruent and incongruent trials. We take the percentage of eye-movements to the distractor on the first quantiles of the

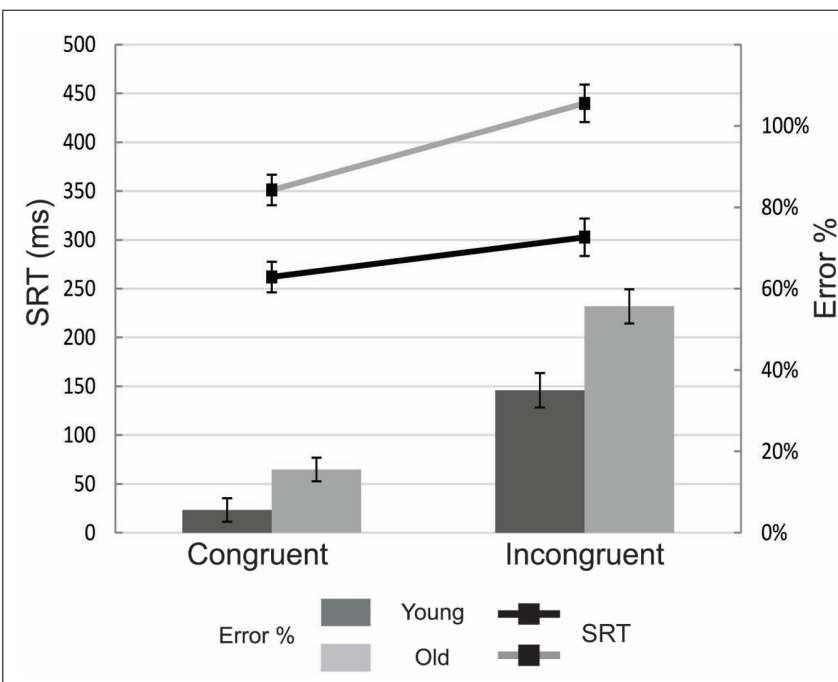

FIGURE 2 | Mean saccade reaction times (SRT; line graph) and error rates (bar graph) for each of the experimental conditions. Young subjects are depicted with black lines and dark-gray bars. Old subjects are depicted with light gray lines and bars. Error bars represent the SEM. 
CCFs to reflect the incidence of oculomotor capture at a stage before top-down control processes have had a chance to develop effective selective suppression of eye-movements to the irrelevant distractor. The percentage of saccades to the distractor on congruent trials starts high and remains high since there is nothing particular in the visual field to attract attention away from the distractor/target, resulting in a relatively flat CCF near the $100 \%$ level. For incongruent trials, we have argued that the initial response capture will be followed by a build-up of selective suppression of the distractor location. For the CCF this means a negative slope toward asymptote, that is, toward the minimum chance of moving to the distractor with increasing SRT. Eye-movements to the distractor rapidly reach an asymptote in the CCF, after which little further performance benefits are gained by responding even slower. As discussed in the introduction, we argue that the build-up speed and strength of selective suppression is visible in the steepness of the negative slope of incongruent trials, and is most pronounced in the slopes of the fast part of the CCF.

\section{Conditional capture: first quantile (bottom-up capture)}

Participants made fewer fast eye-movements to the distractor on incongruent trials compared to congruent trials $[F(1,36)=82.91$, $p<0.001]$, suggesting that target-presence at the location of the distractor influences the amount of eye-movements made to that location even at this early stage, either via bottom-up (target salience) or top-down influences (target relevance to the task). Older participants were more susceptible to capture by the distractor, as reflected in an interaction between congruence and age on this measure $[F(1,36)=20.29, p<0.001]$.

\section{Conditional capture: slopes (top-down inhibition)}

As reactions get slower, fewer saccades go in the direction of the distractor on incongruent trials. This is reflected in an interaction

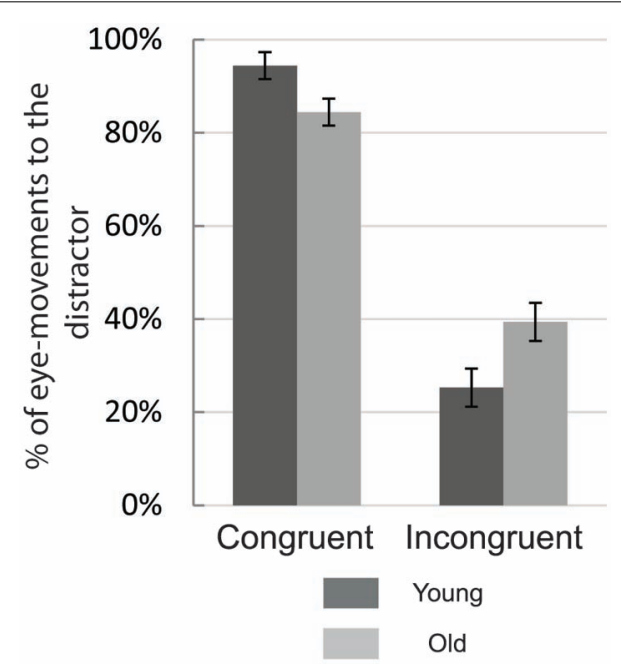

FIGURE 3 | Mean percentage of eye-movements to the distractor for each of the experimental groups. The two left-most bars represent eye-movements to the distractor on congruent trials for young (dark-gray) and old subjects (light gray. The two right-most bars portray incongruent trials. Error bars represent the SEM. between quantile and congruence $[F(3,108)=14.65, p<0.001]$ on CCF slopes, whereby incongruent CCFs decline sharply at first but then level of to an asymptote for the slowest responses. In contrast congruent CCFs slopes remain level from the start as no suppression is required. The decline in capture on incongruent trials with slower SRTs is less pronounced for the older compared to the younger group [quantile $\times$ congruence $\times$ age: $F(3,108)=3.75, p=0.021]$.

An additional ANOVA was conducted focusing exclusively on the slope between the first and second quantiles. This analysis showed the expected effects of congruence $[F(1,36)=98.97$, $p<0.001]$ and a pronounced interaction between congruence and age $[F(1,36)=20.92, p<0.001]$ reflecting the sharp reduction of saccades to the distractor on incongruent trials from the first to the second quantile for the younger group, much more than for the older group.

The difference in these early CCF slopes on incongruent trials between the two groups was slightly enhanced for super-compared to equiluminant distractors [congruence $\times$ age $\times$ distractor luminance: $F(1,36)=4.51, p=0.041]$, such that the effect was significant for superluminant distractors $[F(1,18)=34.97, p<0.001]$, but not for equiluminant distractors $[F(1,18)=2.21, p=0.154]$.

\section{Conditional capture: correlations between first quantile and slope measures}

To examine the extent to which the processes reflected in the first quantile and in the slope of the fastest segment of CCFs (presumably oculomotor capture and oculomotor inhibition, respectively) represent independent processes, we correlated the percentage of eye-movements to the incongruent distractor on the first quantiles of the CCFs with the slopes of the first segment of the CCFs, for each age group separately. These analyses showed that oculomotor capture and its subsequent inhibition

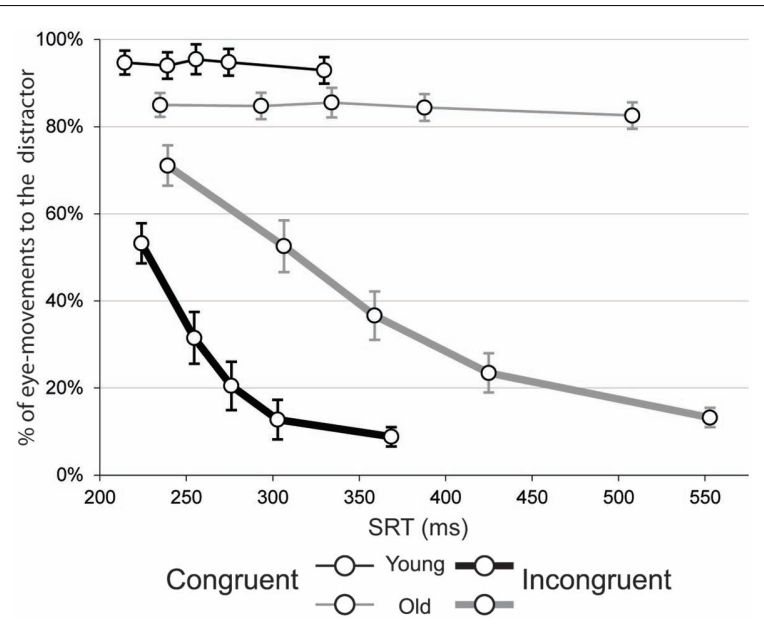

FIGURE 4 | Conditional capture functions (CCFs) for congruent (thin lines) and incongruent (thick lines) trials displaying the percentage of eye-movements toward the distractor ( $y$-axis) plotted against mean saccade reaction time (SRT; $\boldsymbol{x}$-axis) for five speed quantiles. Old subjects are depicted with gray lines, while black lines are used for the young group. Error bars represent the SEM. 
were uncorrelated in both young $[r(20)=-0.219, p=0.354]$ and older adults $[r(20)=-0.309, p=0.186]$.

\section{MANUAL PERFORMANCE}

Replicating Kramer et al. (2000), manual responses we far slower among older than young adults $[F(1,36)=97.05, p<0.001$; see Figure 5]. Target-distractor incongruence also caused slower manual RTs $[F(1,36)=19.95, p<0.001]$, especially so for older adults $[F(1,36)=10.18, p=0.003]$, who traded speed for accuracy and made fewer manual errors in general $[F(1,36)=6.59, p=0.015]$. Such a trade-off was not present for the congruence manipulation, as incongruent trials elicited more manual errors $[F(1,36)=16.65$, $p<0.001]$. Age and congruence did not interact $[F(1,36)=0.35]$.

\section{DISCUSSION}

The present study focused on the influence of normal aging on the tendency to activate automatic eye-movements toward taskirrelevant, salient visual onsets, and the ability to suppress such misdirected saccades. Such age effects have been extensively documented (for a recent example and review see Ryan et al., 2007). Distributional analyses in the form of CCFs were used in an effort to pinpoint in more detail the effects of selective inhibitory control on oculomotor capture.

\section{OVERALL SACCADE AND MANUAL PERFORMANCE}

The overall saccade and performance measures of response speed and accuracy showed that the abrupt-onset distractor strongly interfered with performance on incongruent trials. When target and distractor locations did not coincide, a general deterioration on all dependent measures was seen for both groups: participants were both slower and more error-prone, and this was the case for saccades as well as manual responses. Older adults exhibited slower and less accurate eye-movements. For manual responses a speed-accuracy trade-off was seen as button press responses were

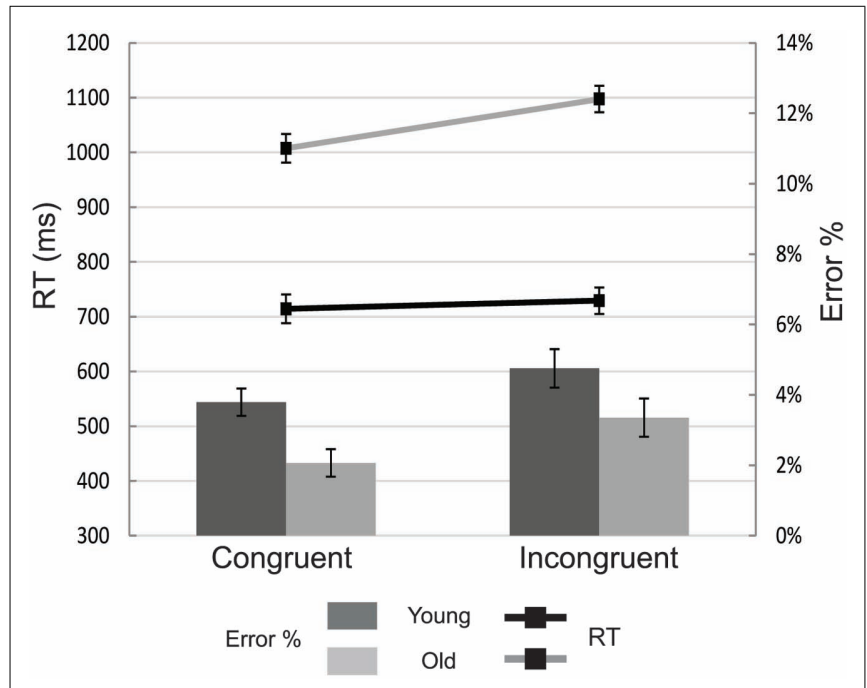

FIGURE 5 | Mean manual reaction times (RT; line graph) and error rates (bar graph) for each of the experimental conditions. Young subjects are depicted with black lines and dark-gray bars. Old subjects are depicted with light gray lines and bars. Error bars represent the SEM. on average as much as $300 \mathrm{~ms}$ slower for the older group. This trade-off rendered the older subjects somewhat more accurate. Because of the speed-accuracy trade-off in manual performance data, and because all hypotheses centered on oculomotor performance, we will focus the discussion on the latter. The irrelevant distractor not appearing at the target location posed more problems for the older compared to younger group as seen in the interaction effects on saccade and manual response speeds.

The effect of congruence on overall saccade accuracy was comparable between young and older participants. At first sight, this pattern seems to discord with the widely observed age changes in distractibility. However, this overall accuracy includes errors in which the eyes move to one of the non-targets (i.e., a circle that was neither distractor nor target). Hence, a more precise measure of oculomotor capture is provided by the percentage of initial eye-movements going to the distractor, in particular on incongruent trials. On congruent trials, targets were contained within the distractor circle and therefore an eye-movement to the target/distractor could either be the result of capture by the distractor, or a deliberate goal-directed movement to the target, or a combination of both. On incongruent trials, an eye-movement to the distractor represents a more unequivocal manifestation of oculomotor capture. Using this measure, older participants were found to be more susceptible to capture my incongruent distractors.

\section{DISTRIBUTIONAL ANALYSES: DIFFERENCES IN CAPTURE OR SUPPRESSION}

The pattern of eye-movements being misdirected to taskirrelevant onsets is consistent with recurrent reports of age-related differences in the susceptibility to distractor effects. However, whether these age changes relate to oculomotor capture or oculomotor inhibition remained to be addressed. Importantly, distributional analyses offer a closer look at the extent to which oculomotor capture and inhibition differ among young and older adults. Agerelated deficiencies in oculomotor capture should be revealed in age effects on the first quantiles of the CCF, as these quantiles represent the fastest eye-movements to the irrelevant distractor, in which the influence of top-down selective response suppression is minimal (Wijnen and Ridderinkhof, 2009). Age-related deficiencies in oculomotor inhibition should be revealed in age effects on the slopes of the CCF. If younger subjects are better able to selectively suppress saccades to the distractor, this will translate to CCFs reaching asymptote faster, and thus steeper slopes between CCF levels at fast and slower quantiles.

The CCFs indicated that our participants were highly susceptible to oculomotor capture by abrupt-onset distractors at incongruent locations, but were able to subsequently suppress the interfering effects of this capture. The percentage of eyemovements to the distractor on the first quantiles of the CCFs shows that older participants were more susceptible to oculomotor capture by the distractor, as reflected in an interaction between congruence and age on this measure. Selective suppression became progressively stronger with slower responses for all participants, although the build-up of suppression was significantly weaker for the older group. The largest differences were expected and seen in the early part of the CCF where the incidence of capture was 
sharply reduced for the young subjects but less so for the older group. Among both young and older adults, these CCF measures were not correlated, suggesting that oculomotor capture and oculomotor inhibition represent stochastically independent processes, and hence that age-related deficiencies in inhibition did not result directly or indirectly from deficiencies in capture: those older individuals who are particularly susceptible to oculomotor capture are not necessarily the same as those who are particularly poor at oculomotor inhibition. However, we must acknowledge that accepting such a null finding might require replication using a more powerful design with more subjects.

The considerable differentiation in movement to incongruent compared to incongruent distractors among young adults might suggest that they are exerting top-down control even in the fastest responses. Note however that even the young start out below chance level; even if they are attempting to suppress oculomotor capture, they are still failing most of the time.

Some mention of the luminance factor is in order. Even though (1) the luminance factor was of little theoretical relevance for present purposes, (2) all participants reported to be well aware of the distractors, regardless of their luminance, and (3) the luminance factor failed to engage in all but one main or interaction effect, in discussing the results we chose to focus on factors more central to current goals. Yet, the one higher-order interaction in which luminance featured was complex but potentially interesting. That is, the age-related differences in congruence effects on early CCF slopes were more pronounced (and significant only) for superluminant distractors. Future studies should determine to what extent age-related decline in the suppression of capture depends on distractor luminance in a more fine-grained analysis.

We should acknowledge a number of potential limitations to this study. First, since oculomotor capture is best expressed in accuracy of the fastest bin, the efficiency of control over such capture can be analyzed as the reduction of such capture in subsequent bins. Of note, the slope measure used here is sensitive to variance in accuracy in the first bin, and hence not entirely independent from the capture measure. One might object though that the more capture there is, the more room there is for subsequent control over capture; hence, the slope measure does carry the relevant information. Yet, as an alternative, one might consider the slope of the segment between the second and third bin, a measure of control that does not entail variance in the capture measure. In principle, however, this slope could be subject to floor effects. Figure 4 suggests that accuracy reaches asymptote for young adults much earlier in the distribution that for older adults. Indeed, ANOVA on the second slope measure yielded an only marginally significant interaction between age and congruency; we cannot be sure whether this lack of statistical significance is due to floor effects or to a true absence of interaction. However, since the second slope measure shows a similar pattern as the first slope, we believe our interpretation is justified.

Second, we concluded that older adults are more susceptible to capture by the distractor; how, then, can we explain that they benefited less from congruent distractors? In using eye-movements tasks with elderly participants, we often note that seniors make more saccadic errors in general (e.g., also in prosaccades in the context of an antisaccade task). So, this seems to reflect a general oculomotor accuracy effect (that are not typically observed in manual RT tasks) - but obviously, we can only speculate. Third, IQ was not measured in this study. Although similar studies typically either do not report specific age effects to disappear after partialling out co-variance with IQ, or do not report IQ at all, we cannot exclude that the age differences observed here could be explained (at least in part) by age differences in general intellectual ability.

\section{RELATION TO OTHER FINDINGS}

The present findings replicate and extend other reports of agerelated problems with oculomotor capture and suppression (e.g., Kramer et al., 2000; Gottlob et al., 2007; Ryan et al., 2007). Findings of deficient inhibition are not reported ubiquitously, however. As posited by Nieuwenhuis et al. (2000), age differences can be observed primarily in tasks that require an active intentional inhibition of prepotent response tendencies. Salient distractors may invoke a more active intentional form of oculomotor inhibition than less salient distractors. Such more active inhibition may rely more on available working-memory capacity. Note that age-related declines in oculomotor inhibition have been related previously to reduced functional working-memory capacity (Eenshuistra et al., 2004). Based on extensive meta-analysis, it has been argued more generally that top-down executive control fails to explain age-related variance in complex cognition over and beyond the effects of working-memory (Verhaeghen, 2011).

We have argued, however, that the specificity of response inhibition is often absorbed by other sources of variance in many tasks often invoked in the study of inhibitory control, and that specific inhibitory effects are easily overlooked when temporal dynamics are ignored (van den Wildenberg et al., 2010). The dopamine-dependent frontostriatal mechanisms underlying these dynamics (for a review see Ridderinkhof et al., 2011) coincide with those deemed to be most crucially involved in cognitive aging (Klostermann et al., 2011). As a central assertion, we contend here that distributional analyses (currently in the form of CCFs) are crucial for an accurate examination of selective inhibitory control over oculomotor capture, and for exposing specific age-related changes in the efficiency of these processes. Applying such analyzes here comprises an innovation beyond approaches used elsewhere (Kramer et al., 2000; Nieuwenhuis et al., 2000; Gottlob et al., 2007; Ryan et al., 2007).

Previous work has demonstrated that deficient performance in the antisaccade task among healthy seniors as well as patients diagnosed with Parkinson's disease could be remedied by motivational incentives such as the prospect of reward (Harsay et al., 2010). These effects of motivational incentives on oculomotor performance likely involve activation and functional connectivity of the dorsal striatum (in particular the nucleus caudatus), which is key to selective oculomotor suppression (Harsay et al., 2011) and specifically sensitive to aging (Klostermann et al., 2011). This pattern would seem to suggest that motivational incentives modulate inhibitory control rather than oculomotor capture, a hypothesis that might be tested using the CCF analyses employed here in the context of healthy aging. 


\section{CONCLUSION}

The present findings confirm that a salient stimulus poses problems for older people when they are asked to ignore that stimulus and suppress any eye-movements toward it, and as such replicate typical findings (e.g., Kramer et al., 2000). Distribution analyses add novel insights to these age changes by showing that they stem from two independent sources: an increase in the bottom-up capture of eye-movements by salient abrupt-onset distractors, and a failure to swiftly and actively suppress such captured oculomotor actions.

In conclusion, the results of the current study suggest that the effects of aging found in the context of salient distractors might

\section{REFERENCES}

Eenshuistra, R. M., Ridderinkhof, K. R., and van der Molen, M. W. (2004). Age-related changes in antisaccade task performance: inhibitory control or working-memory engagement? Brain Cogn. 56, 177-188.

Gottlob, L. R., Fillmore, M. T., and Abroms, B. D. (2007). Age-group differences in inhibiting an oculomotor response. Neuropsychol. Dev. Cogn. B Aging Neuropsychol. Cogn. 14, 586-593.

Harsay, H. A., Buitenweg, J. I. V., Wijnen, J. G., Guerreiro, M. J. S., and Ridderinkhof, K. R. (2010). Remedial efffects of motivational incentive on declining cognitive control in healthy aging and Parkinson's disease. Front. Aging Neurosci. 2:144. doi:10.3389/fnagi.2010. 00144

Harsay, H. A., Cohen, M. X., Oosterhof, N. N., Forstmann, B. U., Mars, R. B., and Ridderinkhof, K. R. (2011). Functional connectivity of the striatum links motivation to action control in humans. J. Neurosci. 31, 10701-10711.

Ishihara, S. (1992). Ishihara's Tests for Colour-Blindness. Tokyo: Kanehara \& Co.

Klostermann, E. C., Braskie, M. N., Landau, S. M., O’Neill, J. P., and Jagust, W. J. (2011). Dopamine and frontostriatal networks in cognitive aging. Neurobiol. Aging. PMID: 21511369. [Epub ahead of print].
Kramer, A. F., Hahn, S., Irwin, D. E., and Theeuwes, J. (1999). Attentional capture and aging: implications for visual search performance and oculomotor control. Psychol. Aging 14, 135-154.

Kramer, A. F., Hahn, S., Irwin, D. E., and Theeuwes, J. (2000). Age differences in the control of looking behavior: do you know where your eyes have been? Psychol. Sci. 11, 210-217.

McCormick, P. A. (1997). Orienting attention without awareness. J. Exp. Psychol. Hum. Percept. Perform. 23, 168-180.

Nieuwenhuis, S., Ridderinkhof, K. R., de Jong, R., Kok, A., and van der Molen, M. W. (2000). Inhibitory inefficiency and failures of intention activation: age-related decline in the control of saccadic eye movements. Psychol. Aging 15, 635-647. ment in the ability to ignore irrelevant information. J. Gerontol. 20, 233-238.

Ridderinkhof, K. R., Forstmann, B. U., Wylie, S. A., Burle, B., and van den Wildenberg, W. P. M. (2011). Neurocognitive mechanisms of action control: resisting the call of the Sirens. Wiley Interdiscip. Rev. Cogn. Sci. 2, 174-192.

Ryan, J. D., Leung, G., Turk-Browne, N. B., and Hasher, L. (2007). Assessment of age-related changes in inhibition and binding using
Rabbitt, P. M. A. (1965). An age decre-

be explained by stronger capture of saccades and (independently) weaker selective suppression of such oculomotor tendencies. The fact that these process-specific age effects remained concealed in overall oculomotor performance analyses underscores once more the utility of distributional analyses to look beyond the surface.

\section{ACKNOWLEDGMENTS}

The present study was supported by grants from the Netherlands Organization for Scientific Research (NWO) to K. Richard Ridderinkhof. The authors wish to thank Marcus Spaan for technical assistance and Sanne Koemans for suggestions relating to the experimental task and help in collecting the data.

eye-movement monitoring. Psychol. Aging 22, 239-250.

Stürmer, B., Leuthold, H., Soetens, E., Schröter, E., and Sommer, W. (2002). Control over location-based response activation in the Simon task: behavioral and electrophysiological evidence. J. Exp. Psychol. Hum. Percept. Perform. 28, 1345-1363.

Theeuwes, J., Kramer, A. F., Hahn, S., and Irwin, D. E. (1998). Our eyes do not always go where we want them to go: capture of the eyes by new objects. Psychol. Sci. 9, 379-385.

van den Wildenberg, W. P. M., Wylie, S. A., Forstmann, B. U., Burle, B., Hasbroucq, T., and Ridderinkhof, K. R. (2010). To head or to heed: beyond the surface of selective action inhibition. Front. Neurosci. 4:222. doi:10.3389/fnhum.2010.00222

Verhaeghen, P. (2011). Aging and executive control: reports of a demise greatly exaggerated. Curr. Dir. Psychol. Sci. 20, 174-180.

Vincent, S. B. (1912). The function of the vibrissae in the behavior of the white rat. Behav. Monogr. 1, $1-82$.

Wijnen, J. G., and Ridderinkhof, K. R. (2007). Response inhibition in motor and oculomotor conflict tasks: different mechanisms, different dynamics? Brain Cogn. 63, 260-270.

Wijnen, J. G., and Ridderinkhof, K. R. (2009). Contextual modulation of inhibitory oculomotor control reflected in $\mathrm{N} 2$ and saccade reaction time distributions. Psychophysiology 46, 1-10.

Zacks, R., and Hasher, L. (1997). Cognitive gerontology and attentional inhibition: a reply to Burke and McDowd. J. Gerontol. B Psychol. Sci. Soc. Sci. 52, 274-283.

Conflict of Interest Statement: The authors declare that the research was conducted in the absence of any commercial or financial relationships that could be construed as a potential conflict of interest.

Received: 29 April 2011; paper pending published: 07 June 2011; accepted: 26 September 2011; published online: 25 October 2011.

Citation: Ridderinkhof KR and Wijnen JG (2011) More than meets the eye: age differences in the capture and suppression of oculomotor action. Front. Psychology 2:267. doi: 10.3389/fpsyg.2011.00267

This article was submitted to Frontiers in Cognition, a specialty of Frontiers in Psychology.

Copyright (C) 2011 Ridderinkhof and Wijnen. This is an open-access article subject to a non-exclusive license between the authors and Frontiers Media SA, which permits use, distribution and reproduction in other forums, provided the original authors and source are credited and other Frontiers conditions are complied with. 